\title{
Perception du changement climatique par les éleveurs de bovins des zones tropicales sèche et subhumide du Bénin : comparaison avec les données météorologiques
}

\author{
Yaya Idrissou $^{1, *}$, Alassan Assani Seidou ${ }^{1}$, Fréjus Mahougnon Tossou ${ }^{1}$, Hilaire Sorébou Sanni Worogo ${ }^{1}$, \\ Mohamed Nasser Baco ${ }^{2}$, Josias Steve Adjassin ${ }^{1}$, Brice Gérard Comlan Assogba ${ }^{1}$ \\ et Ibrahim Alkoiret Traore ${ }^{1}$ \\ ${ }^{1}$ Laboratoire d'Écologie, Santé et Production Animales (LESPA), Faculté d'Agronomie (FA), Université de Parakou, 01 BP 123 , \\ Parakou, République du Bénin \\ ${ }^{2}$ Laboratoire Société-Environnement (LaSEn), Faculté d'Agronomie, Université de Parakou, 01 BP 123, Parakou, République du Bénin
}

\begin{abstract}
Résumé - Une fausse perception du changement climatique peut entraîner une absence d'adaptation ou une mauvaise adaptation, augmentant ainsi la vulnérabilité à ce changement. Cependant très peu d'études se sont intéressées à cette question, surtout chez les éleveurs. Cette étude compare la perception du changement climatique des éleveurs de bovins des zones tropicales sèche et subhumide du Bénin avec les données climatiques des 40 dernières années. À cet effet, 360 éleveurs de bovins ont été interrogés dans ces zones. Les données collectées sont relatives aux caractéristiques sociodémographiques des éleveurs, ainsi qu'à leur perception du changement climatique. Les données climatiques couvrant la période 1976-2015 ont été collectées dans les stations météorologiques les plus proches de chaque zone d'étude. Les données d'enquête ont été soumises aux analyses fréquentielles et les données climatiques aux anomalies standardisées. Les résultats montrent que plus de $70 \%$ des éleveurs des deux zones d'étude ont perçu une installation plus tardive de la saison pluvieuse, ainsi qu'une augmentation de la température et de la durée des poches de sécheresse, ce qui correspond aux données climatiques. Par contre, il existe un décalage entre les données climatiques et les perceptions des éleveurs, pour lesquels la pluviométrie aurait diminué et la saison des pluies se terminerait plus tôt. Cette étude permet de conclure que l'intégration d'indicateurs du changement perçus par les éleveurs serait pertinente pour élaborer des stratégies d'adaptation au changement climatique appropriées, consensuelles et durables, en facilitant la compréhension et la concertation entre éleveurs et scientifiques.
\end{abstract}

Mots clés : éleveurs / bovin / changement climatique / perceptions / Bénin

\begin{abstract}
Perception of climate change by cattle farmers in dry and sub-humid tropical zones of Benin: comparison with meteorological data. A false perception of climate change can lead to lack of adaptation or maladaptation, thus increasing vulnerability to climate change. Nevertheless, very few studies have focused on this question, especially with cattle breeders. This study compares the climate change perception of cattle breeders in the dry and sub-humid tropical zones of Benin with the climatic data of the last 40 years. For this purpose, 360 cattle farmers were surveyed in these areas. The data collected were related to the socio-demographic characteristics of the farmers, as well as their perception of climate change. In addition, climatic data covering the period 1976-2015 were collected in the meteorological stations closest to each study area. Survey data were processed through frequency analysis and climate data through standardized anomalies. The results show that more than $70 \%$ of farmers in both study areas perceived a later installation of the rainy season, and an increase in temperature and pockets of drought, which is consistent with climate data. Nevertheless, there is a disagreement between climate data and farmers' perceptions on rainfall decrease and earlier ending of the rainy season. This study allows us to conclude that the integration of indicators of change perceived by cattle farmers would be relevant to develop appropriate
\end{abstract}

\footnotetext{
*Auteur de correspondance : yayaidriss2617@gmail.com
} 
climate change adaptation strategies, consensual and sustainable. It will facilitate understanding and dialogue between farmers and scientists.

Keywords: cattle farmers / cattle / climate change / perception / Benin

\section{Introduction}

Le changement climatique constitue un obstacle au développement socioéconomique des populations rurales (Niang, 2009). À l'échelle planétaire, le changement climatique induit une élévation de la température et une nouvelle répartition des précipitations (Bergonzini, 2004). Dans ce contexte mondial, l'Afrique subsaharienne, où l'agriculture constitue la principale source d'emploi et de revenus pour la majorité de la population (Enete et Onyekuru, 2011), apparaît comme la région du monde la plus exposée au changement climatique (FAO, 2008).

Le Bénin, pays en développement d'Afrique subsaharienne, n'est pas à l'abri des menaces liées au changement climatique. Selon le ministère de l'Environnement, de l'Habitat et de l'Urbanisme (MEHU, 2011), les précipitations resteront plus ou moins stables $(+0,2 \%)$ dans le Sud du pays, mais seront réduites de 13 à $15 \%$ dans le Nord à l'horizon 2100. Avec ces scénarios, les élevages bovins installés dans le nord du Bénin, zone de prédilection de l'élevage bovin du pays (Djènontin, 2010), et fortement dépendants des ressources pastorales spontanées, subiront fortement les effets des variations climatiques, avec pour corollaire des baisses de productivité du bétail (Nardone et al., 2010). Néanmoins, le changement climatique pourrait aussi avoir un effet bénéfique sur la productivité des parcours et prairies à travers l'augmentation de la photosynthèse permise par l'augmentation du $\mathrm{CO}_{2}$ atmosphérique (Graux, 2011).

Des études ont apporté des éclaircissements sur les perceptions des éleveurs face au changement climatique (Dossa et al., 2017). Cependant, le lien entre ces perceptions et les données météorologiques n'a pas souvent été analysé. Le peu de travaux existant sur le sujet se sont focalisés sur les agriculteurs et agro-éleveurs (Bambara et al., 2016; Ayanlade et al., 2017), puis généralisent les résultats obtenus aussi bien aux agriculteurs, agro-éleveurs qu'aux éleveurs de bovins; or ces catégories socio-professionnelles n'ont pas les mêmes contraintes (Zampaligré et al., 2013). En effet, pour assurer l'alimentation des animaux, les éleveurs de bovins ont largement recours aux parcours naturels, contrairement aux agro-éleveurs et agriculteurs. Ainsi, une étude permettant de comparer la perception des éleveurs aux données météorologiques s'avère nécessaire, dans la mesure où elle peut contribuer à mieux éclairer et orienter les stratégies spécifiques d'adaptation (Jarawura, 2014). La présente étude a pour objectif d'analyser la cohérence des perceptions du changement climatique par les éleveurs de bovins des zones tropicales sèche et subhumide du Bénin avec les données météorologiques des quarante dernières années.

\section{Matériel et méthodes}

\subsection{Milieu d'étude}

L'étude a été conduite dans deux zones climatiques du Bénin : la zone tropicale sèche et la zone tropicale subhumide, situées au Nord du pays. Le choix de ces zones repose sur le fait que les prévisions climatiques indiquent qu'elles sont les plus vulnérables au déficit pluviométrique (Gnanglè et al., 2011; MEHU, 2011). De plus, le cheptel bovin est concentré à plus de $85 \%$ dans ces zones (Alkoiret et al., 2011). Dans chaque zone, six villages répartis sur deux communes ont été choisis sur la base de l'importance de l'effectif des bovins et d'entretiens préliminaires avec les techniciens locaux. Ainsi, les communes de Tchaourou et Nikki ont été retenues dans la zone tropicale subhumide et ceux de Gogounou et Banikoara dans la zone tropicale sèche (Fig. 1).

\section{Collecte des données}

\subsection{Données primaires}

La collecte des données primaires (caractéristiques sociodémographiques et perceptions du changement climatique par les éleveurs) a été faite de juin à novembre 2018 en deux étapes: la phase exploratoire et la phase approfondie.

\subsubsection{Enquête exploratoire}

L'enquête exploratoire a été réalisée en trois étapes que sont: entretien avec les techniciens locaux, identification des villages à étudier et identification des éleveurs.

\subsubsection{Entretiens avec les techniciens locaux}

Des entretiens ont été effectués avec les techniciens des Agences territoriales pour le développement agricole afin d'identifier les villages à étudier. Les critères de choix des villages étaient l'accessibilité et le nombre d'éleveurs de bovins dans le village (supérieur à 30).

\subsubsection{Identification des villages}

Sur la base des critères définis, avec les techniciens locaux, les villages de Koubou, Papanè et Agbassa ont été identifiés dans la commune de Tchaourou; les villages de Tèbo, Biro et Sakabansi dans la commune de Nikki; les villages de Bagou, Fana et Lougou dans la commune de Gogounou et enfin ceux de Founougo, Goumori et Soroko dans la commune de Banikoara (Fig. 1).

\subsubsection{Identification des éleveurs}

Nous avons identifié 360 éleveurs de bovins, soit 30 éleveurs par village. Nous les avons sélectionnés au hasard parmi les éleveurs qui répondaient aux deux critères suivants : avoir l'élevage bovin comme activité principale et être âgé d'au moins 50 ans. Le choix de l'élevage comme activité principale pour discriminer les personnes interrogées se justifie par le fait que plusieurs études se focalisent sur les agro-éleveurs et généralisent les résultats obtenus aussi bien aux agro-éleveurs qu'aux éleveurs de bovins ; or ces catégories socio-professionnelles sont confrontées à des problèmes 


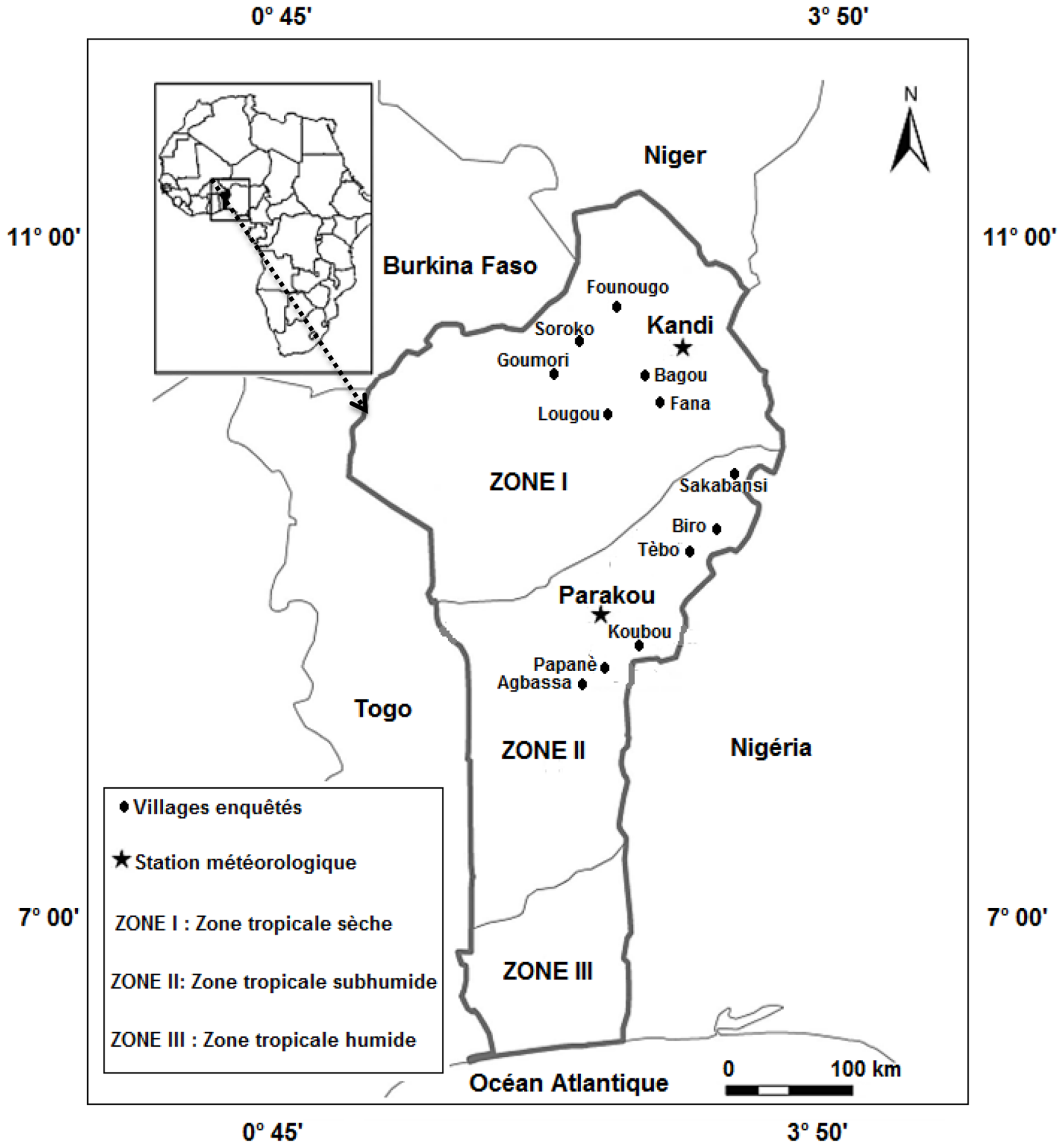

Fig. 1. Localisation des villages et des stations météorologiques étudiés au Bénin.

Fig. 1. Location of villages and meteorological stations studied in Benin.

socio-économiques différents (Zampaligré et al., 2013). La barrière d'âge (50 ans) s'explique par le fait que nous voulions remonter 40 ans en arrière pour analyser les faits liés au changement climatique.

\subsubsection{Enquête approfondie}

La phase d'enquête approfondie a consisté en une collecte des données à travers des entretiens semi-structurés
(Encadré 1) auprès des 360 éleveurs identifiés lors de l'enquête exploratoire. Les principales données collectées auprès des éleveurs concernent leurs caractéristiques sociodémographiques (sexe, âge, ethnie, taille du ménage, niveau d'instruction, expérience en élevage, contact avec la vulgarisation, maind'œuvre employée et appartenance à une organisation) et leurs perceptions du changement climatique concernant la pluviométrie et la température. Le questionnaire a été testé lors des focus group de 6 à 15 éleveurs, à raison d'un focus group par village. 
Encadré 1. Quelques éléments clés du guide d'entretien relatif à l'étude de la perception du changement climatique par les éleveurs de bovins des zones tropicales sèches et subhumide du Bénin.

1. Qu'est-ce qui vous vient à l'esprit lorsqu'on parle du changement climatique?

$\begin{array}{ll}\text { Sujet de réponse possible } & \text { Source de l'information } \\ & \text { Causes } \\ & \text { Conséquences }\end{array}$

2. Avez-vous observé autour de vous des évènements ou des évolutions qui seraient liés au changement climatique ces dernières années?

3. Quelles sont les types du changement climatique perçus?

4. Que pensez-vous de la pluviométrie depuis 40 ans?

Sujet de réponse possible Augmentée

Diminuée

Aucun changement

Ne sait pas

5. Que pensez-vous de la température depuis 40 ans?

Sujet de réponse possible

Augmentée

Diminuée

Aucun changement

Ne sait pas

6. Que pensez-vous du début de la saison des pluies ces 40 dernières années?

Sujet de réponse possible Tôt

Tard

Aucun changement

$\mathrm{Ne}$ sait pas

7. Que pensez-vous de la fin de la saison des pluies ces 40 dernières années?

Sujet de réponse possible Tôt

Tard

Aucun changement

Ne sait pas

8. Que pensez-vous de la durée des poches de sécheresse ces 40 dernières années?

Sujet de réponse possible Augmentée

Diminuée

Aucun changement

Ne sait pas

\subsection{Données secondaires}

Afin de confronter la perception des éleveurs à l'évolution réelle des paramètres climatiques, des données climatiques (température et précipitations) couvrant les 40 dernières années (1976-2015) de la station météorologique la plus proche de chaque zone d'étude ont été collectées. Ainsi les données de la station météorologique de Kandi ont été utilisées pour la zone tropicale sèche et celles de Parakou pour la zone tropicale subhumide.

\subsection{Analyse des données}

\subsubsection{Données primaires}

Après dépouillement, les données des enquêtes ont été traitées à l'aide du logiciel SPSS. Nous avons choisi le code 1 pour les réponses affirmatives sur la perception d'un indicateur climatique et le code 0 pour les réponses négatives. Les réponses (taux de perception) ont été traduites sous forme de proportions centésimales, puis comparées par le test de Chicarré $\left(\chi^{2}\right)$. Lorsque le test $\chi^{2}$ a indiqué des relations significatives, un test post-hoc a été réalisé à l'aide de la méthode des résidus standardisés. Les variables quantitatives décrivant les éleveurs de bovins interrogés ont été présentées sous forme de moyennes \pm écarts-types, et comparées entre les zones à l'aide du test non paramétrique $U$ de Mann-Whitney (McDonald, 2009).

\subsubsection{Données secondaires}

La hauteur pluviométrique, le début et la fin de la saison pluvieuse, la durée des poches de sécheresse et les températures maximales sont les facteurs du climat qui ont fait objet d'analyses dans cette étude. Le début et la fin de la saison des pluies au cours de chaque année de la période d'étude ont été déterminés suivant l'approche de Sivakumar et al. (1993). La durée des poches de sécheresse a été déterminée par l'évolution des écarts (exprimés en nombre de jours) les plus élevés entre deux pluies consécutives durant les mois de pleine saison des pluies (Ibrahim et al., 2012). Une poche de sécheresse, encore appelée séquence sèche ou pause pluviométrique, est un épisode de manque d'eau plus ou moins long séparant deux pluies et pouvant affecter la flore et les sols. Une poche de sécheresse intervient au cours de la saison des 
Tableau 1. Caractéristiques sociodémographiques des éleveurs interrogés.

Table 1. Socio-demographic characteristics of the interviewed cattle farmers.

\begin{tabular}{|c|c|c|c|c|c|}
\hline \multirow[b]{2}{*}{ Variables } & \multirow[b]{2}{*}{ Total } & \multicolumn{2}{|c|}{ Zone climatique } & \multirow[b]{2}{*}{$\chi^{2}$} & \multirow[b]{2}{*}{$p$-value } \\
\hline & & Zone tropicale subhumide & Zone tropicale sèche & & \\
\hline \multicolumn{6}{|l|}{ Pourcentage $(\%)$} \\
\hline Masculin & 92 & 95 & 90 & & \\
\hline Féminin & 8 & 5 & 10 & & \\
\hline \multicolumn{6}{|l|}{ Ethnie } \\
\hline Bariba & 20 & 20 & 18 & & \\
\hline \multicolumn{6}{|l|}{ Niveau d'instruction } \\
\hline Scolarisé & 5 & 7 & 3 & & \\
\hline Non scolarisé & 95 & 93 & 97 & 2,10 & 0,14 \\
\hline Appartenance à une organisation & 90 & 88 & 94 & 2,19 & 0,13 \\
\hline Contact avec la vulgarisation & 66 & $61^{\mathrm{a}}$ & $72^{\mathrm{b}}$ & 4,95 & 0,02 \\
\hline \multicolumn{6}{|l|}{ Moyenne \pm écart type } \\
\hline Nombre actifs agricole (personne) & $7 \pm 3$ & $8 \pm 3$ & $7 \pm 3$ & - & 0,07 \\
\hline Taille du ménage (personne) & $11 \pm 4$ & $11 \pm 4$ & $11 \pm 3$ & - & 0,52 \\
\hline Taille du cheptel bovin (tête) & $64 \pm 38$ & $61 \pm 41$ & $67 \pm 35$ & - & 0,12 \\
\hline
\end{tabular}

a,b : les valeurs de la même ligne indicées de différentes lettres sont significativement différentes au seuil de $5 \%$.

pluies tandis que la sécheresse intervient pendant la saison sèche. Le logiciel Instat+ (Stern et al., 2006) a été utilisé pour effectuer ces différents calculs.

L'évolution des différents paramètres climatiques a été analysée à l'aide de la méthode des indices ou des anomalies centrées réduites (Lamb, 1982) selon la formule:

$$
A s=\frac{(X i-X)}{S},
$$

Où : $A s=$ anomalie centrée réduite $; X=$ module annuel de la variable enregistrée au cours de l'année $i, X=$ moyenne interannuelle de la variable sur la période d'étude, $S=$ écarttype de la série sur la période d'étude.

Les données météorologiques ont été confrontées aux perceptions des éleveurs en vue d'apprécier la concordance de ces informations. Il y a concordance lorsque les données météorologiques ne contredisent pas la perception locale.

\section{Résultats}

\subsection{Caractéristiques sociodémographiques des éleveurs de bovins enquêtés}

Les caractéristiques sociodémographiques des éleveurs interrogés sont résumées dans le tableau 1. La quasi-totalité des éleveurs interrogés sont des hommes $(92 \%)$, en majorité du groupe socioculturel peulh $(80 \%)$. Leur âge varie de 50 à 76 ans ( 56 ans en moyenne) et ils sont à $95 \%$ non scolarisés. La taille du ménage est en moyenne de 11 personnes, celle du cheptel bovin est en moyenne de 64 têtes. L'expérience en élevage des bovins est en moyenne de 30 ans. Un grand nombre d'éleveurs sont membres d'une organisation (90\%) et en contact avec les services de vulgarisation agricole (66\%).

\subsection{Comparaison de la perception des éleveurs aux données météorologiques}

\subsubsection{Pluviométrie et température}

Le tableau 2 présente les taux de perception des éleveurs de bovins des deux zones d'étude sur les changements des régimes de pluviométrie et de température. La majorité des éleveurs de la zone tropicale subhumide $(77 \%)$ et de la zone tropicale sèche $(82 \%)$ ont perçu une diminution de la pluviométrie au cours des 40 dernières années. Ces taux n'ont pas été significativement différents $(p>0,05)$ d'une zone climatique à l'autre. Une comparaison de la perception des éleveurs des deux zones climatiques avec les données climatiques enregistrées (Fig. 2a) a montré qu'il n'existait aucune preuve tangible de la réduction des précipitations en termes de quantité, comme le prétendent la majorité des éleveurs. Au contraire, la pluviométrie a plutôt augmenté dans les deux zones (mais non significatif dans la zone subhumide). Cette augmentation a été plus importante dans la zone tropicale sèche que dans la zone subhumide.

Parmi les éleveurs interrogés, une large majorité, $85 \%$ dans la zone tropicale sèche et $75 \%$ dans la zone tropicale subhumide, ont perçu une augmentation de la température au cours des 40 dernières années (Tab. 2). Une différence significative $(p<0,05)$ a été observée entre ces taux. En comparant cette perception avec les tendances réelles des températures maximales enregistrées au cours des 40 dernières années (Fig. 2b), une nette augmentation a été observée, 
Tableau 2. Perception du changement climatique par les éleveurs de bovins des zones tropicales sèche et subhumide du Bénin. Table 2. Perception of climate change by cattle farmers in dry and sub-humid tropical zones of Benin.

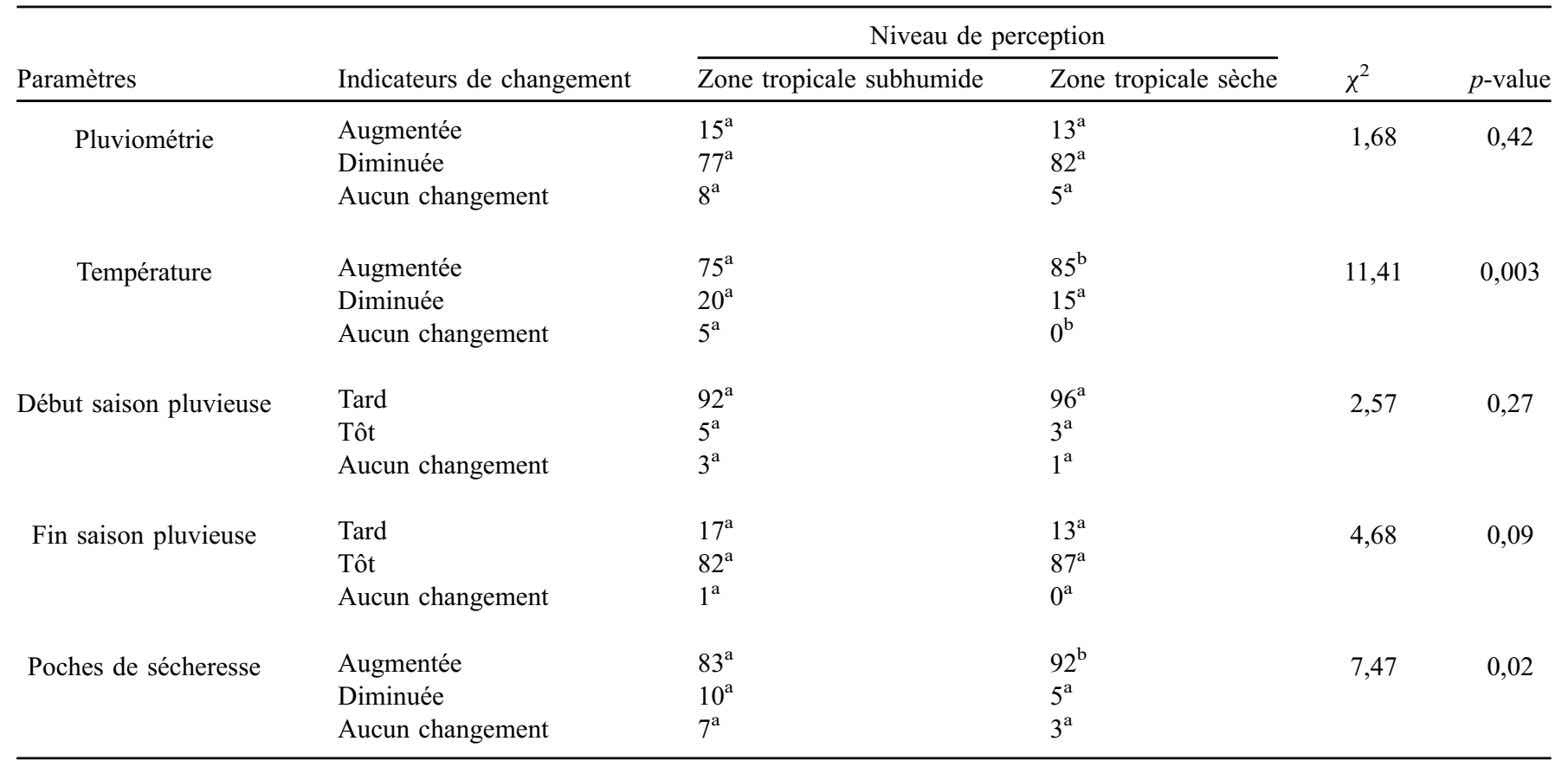

a,b : les valeurs de la même ligne indicées de différentes lettres sont significativement différentes au seuil de $5 \%$.

suggérant que les données climatiques corroborent la perception des éleveurs concernant les changements de température à long terme.

\subsubsection{Début et fin des saisons pluvieuses}

Les éleveurs interrogés ont porté une attention particulière aux caractéristiques de la saison des pluies au fil du temps. La grande majorité des éleveurs dans la zone tropicale sèche (96\%) et dans la zone tropicale subhumide (92\%), estiment qu'aujourd'hui l'installation de la saison pluvieuse est plus tardive (Tab. 2). Par ailleurs, $87 \%$ des éleveurs de la zone tropicale sèche et $82 \%$ des éleveurs de la zone tropicale subhumide ont indiqué que les dates de fin de la saison pluvieuse étaient désormais plus précoces (Tab. 2). Aucune différence significative $(p>0,05)$ n'a été observée entre ces taux. D'après les données climatiques, le début de la saison des pluies dans la zone tropicale sèche montre un recul au cours des 40 dernières années $(p<0,05)$, ce qui montre que la saison des pluies commence plus tard dans cette zone (Fig. 2c). Par ailleurs, même si la saison des pluies a été retardée dans la zone tropicale subhumide au cours des 40 dernières années (Fig. 2c), ce retard n'est pas significatif $(p>0,05)$. Il existe donc un accord entre les données climatiques et la perception des éleveurs des deux zones d'étude concernant les dates plus tardives d'installation de la saison des pluies. En ce qui concerne la date de fin des pluies, la majorité des éleveurs interrogés dans les deux zones ont indiqué que la saison des pluies se termine plus tôt (Tab. 2), alors que les tendances de l'anomalie de fin de saison montrent plutôt une fin de saison plus tardive (Fig. 2d). Il y a donc une contradiction entre les données climatiques et la perception des éleveurs concernant les dates de fin de la saison des pluies.

\subsubsection{Poches de sécheresse}

L'augmentation de la durée des poches de sécheresse en pleine saison pluvieuse a été perçue par un grand nombre d'éleveurs des deux zones climatiques au fil de ces quarante dernières années (Tab. 2). Mais cette perception est plus élevée $(p<0,05)$ dans la zone tropicale sèche $(92 \%)$ que dans la zone tropicale subhumide $(83 \%)$.

La figure 2e montre une augmentation $(p<0,001)$ de la durée des poches de sécheresse aussi bien dans la zone tropicale subhumide que dans la zone sèche. Ce qui confirme la perception des éleveurs.

\section{Discussion}

La perception des éleveurs vis-à-vis du changement climatique n'est pas systématiquement concordante avec les tendances climatiques calculées à partir des relevés d'observations des stations météorologiques. Comme tous les éleveurs des pays en développement (Feleke et al., 2016), la majorité des éleveurs des zones tropicales sèche et subhumide du Bénin ont observé une modification du climat. Les répondants ont observé une augmentation de la température et une diminution de la pluviométrie durant ces 40 dernières années. La perception qu'ont les éleveurs de l'augmentation de la température dans les deux zones d'étude correspond aux données climatiques observées. En effet, la température étant un ressenti direct, l'éleveur peut facilement constater son augmentation à travers des nuits plus chaudes. Par ailleurs, une forte température pousse les animaux à chercher davantage l'ombre et l'eau. Ce comportement des animaux observé par les éleveurs pourrait aussi expliquer l'accord entre leur perception et les données météorologiques. Des résultats 

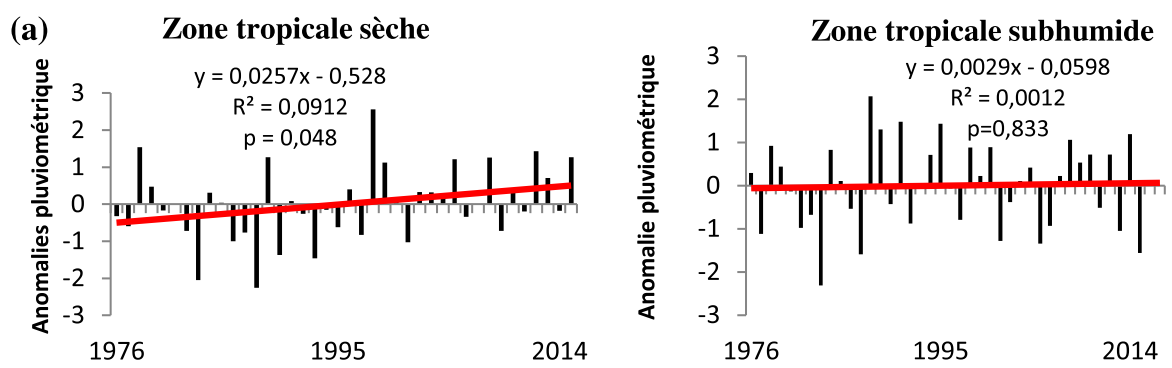

(b)
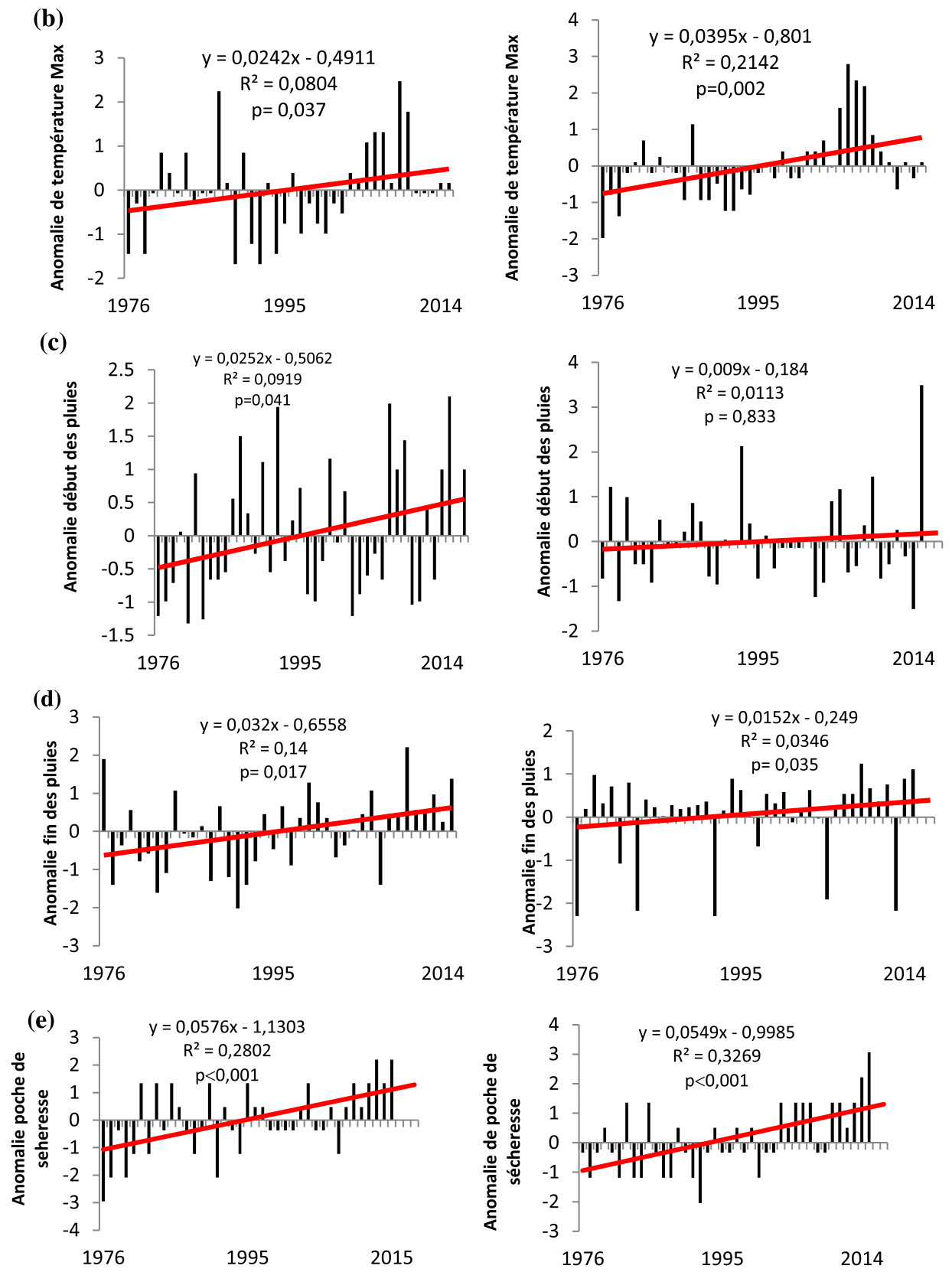

Fig. 2. Anomalies standardisées a : de la pluviométrie ; b : de la température maximale; $\mathrm{c}$ : du début de la saison pluvieuse; : de la fin de la saison pluvieuse; e : et des poches de sècheresse des zones tropicales sèche (à gauche) et subhumide (à droite) du Bénin de 1976 à 2015. Fig. 2. Standardized anomalies a: of rainfall; $b$ : maximum temperature; $c$ : onset of rainy season; $d$ : end of rainy season; e: and pockets of drought in dry (left) and sub-humid (right) tropical zones of Benin from 1976 to 2015. 
similaires ont été rapportés par Hasan et Kumar (2019) au Bangladesh, en ce qui concerne la perception de l'augmentation de la température par les éleveurs. Par contre, CuniSanchez et al. (2018) ont rapporté que les pasteurs du Mont Nyiro au Kenya n'ont observé aucune modification de la température. La différence entre nos résultats et ceux de ces auteurs pourrait être due au type de climat qui règne dans chaque zone d'étude.

La perception qu'ont les éleveurs de la diminution de la pluviométrie au cours des 40 dernières années diverge avec les résultats de l'analyse des données de précipitations observées au cours de ladite période. En effet, la pluviométrie est le résultat de nombreux facteurs (début et fin de la saison des pluies, fréquence des pluies, hauteur des pluies, etc.) et un cumul ne constitue pas un événement facilement perceptible. Les éleveurs des deux zones d'étude perçoivent un certain dérèglement pluviométrique, mais qui ne correspond pas forcément à ce qu'ils traduisent par la «diminution de la pluviométrie». Ce résultat corrobore ceux de Vall et Lankoande (2009), qui ont rapporté que les perceptions des éleveurs de Koumbia et Douroula au Burkina contredisent les tendances climatiques. Ces auteurs ont ainsi abouti à la conclusion que les éleveurs perçoivent le changement climatique à travers la modification de la répartition des pluies et non pas à travers les hauteurs totales de pluies. Selon Ovuka et Lindqvist (2000), la discordance entre les données météorologiques et la perception des éleveurs pourrait s'expliquer par le fait que les scientifiques analysent souvent les données météorologiques à des échelles de temps différentes des échelles prises en compte par les éleveurs. Pour ces derniers, les indicateurs utilisés pour apprécier les paramètres climatiques sont, entre autres, les hauteurs d'eau dans les mares, l'état de la végétation à une date donnée et l'état des animaux (Vall et Diallo, 2009).

L'analyse des caractéristiques de la saison des pluies (date de début, date de fin, poches de sécheresse et durée de la saison des pluies) est pertinente pour les éleveurs, car ce sont des facteurs clés dans la planification des activités pendant la saison (gestion des naissances, pâturage des repousses, etc.). La majorité des éleveurs des deux zones d'étude s'accordent sur le fait que la saison des pluies commence plus tard et se termine plus tôt au cours des quarante dernières années. Les données météorologiques ont montré que, même si la saison des pluies débute plus tardivement, elle ne se termine pas plus tôt comme l'ont affirmé la majorité des éleveurs. Le début de la saison des pluies (Gataaje en fulfulde, la langue des Peulhs) est une période critique pour les éleveurs, car caractérisée par le retour des pluies, la repousse de l'herbe et les premières mise-bas. Pendant cette période, les éleveurs scrutent le ciel pour savoir si l'herbe va bien pousser, et si les vaches à terme vont supporter les premiers mois de lactation. Ceci pourrait expliquer l'accord entre les perceptions et les observations. À la fin de la saison des pluies (Yaamde en fulfulde), il y a moins d'enjeux qu'en début de saison des pluies, hormis l'évitement des dégâts sur les champs cultivés qui arrivent à maturité et l'attente des premières récoltes pour disposer des vaines pâtures. À cette période de fin de la saison des pluies, les difficultés pour les éleveurs proviennent de l'extension des cultures car ils ne savent plus trop où conduire leur troupeau pour le faire pâturer et éviter les conflits avec les agriculteurs. Leur perception d'une fin précoce des pluies (ne correspondant pas aux données climatiques), pourrait traduire cette difficulté.

La perception qu'ont les éleveurs de l'augmentation de la durée des poches de sécheresse au cours des 40 dernières années est en accord avec les données météorologiques. Pourtant, les éleveurs sont moins concernés par les poches de sécheresse que les agriculteurs. Cependant, de plus en plus d'éleveurs s'adonnent à l'agriculture, ce qui pourrait expliquer pourquoi ils attachent de l'importance à ce critère. Ce résultat est en accord avec ceux de Bambara et al. (2016). La perception des éleveurs est donc partiellement conforme aux évolutions climatiques réelles, comme l'a aussi constaté Jarawura (2014).

\section{Conclusion}

Les résultats de cette étude montrent que les éleveurs des zones tropicales sèche et subhumide du Bénin sont conscients du changement climatique. Leurs perceptions sur le changement climatique concordent avec les données de température, de début de saison des pluies et de durée des poches de sécheresse observées. En revanche, elles diffèrent des données de cumul de pluviométrie et de fin de la saison des pluies. Sur la base de ces résultats, les variables température, début de saison des pluies et poches de sécheresse seraient celles à retenir pour discuter du changement climatique avec les éleveurs dans les territoires étudiés.

Remerciements. L'auteur principal remercie la Fondation Internationale pour la Science pour son aide financière (IFS, B/6189-1). Il remercie également le Groupe d'experts intergouvernemental sur l'évolution du climat (GIEC) et la Fondation Prince Albert II de Monaco (FPAM) pour leur soutien financier. Le contenu de cet article n'engage que la responsabilité des auteurs et ne peut en aucun cas être considéré comme un reflet de la position du FPAM ou du GIEC. Les auteurs remercient enfin les experts anonymes qui ont évalué ce manuscrit.

\section{Références}

Alkoiret TI, Radji M, Babatoundé S. 2011. Typologie des élevages bovins installés dans la commune de Ouaké au Nord-Ouest du Bénin. Livestock Research for Rural Development 23: 3.

Ayanlade A, Radeny M, Morton JF. 2017. Comparing smallholder farmers' perception of climate change with meteorological data: A case study from southwestern Nigeria. Weather and Climate Extremes 15: 24-33. DOI: 10.1016/j.wace.2016.12.001.

Bambara D, Thiombiano A, Hien V. 2016. Changements climatiques en zones nord-soudanienne et Sub-sahélienne du Burkina Faso: comparaison entre savoirs paysans et connaissances scientifiques. Revue d'Écologie (Terre et Vie) 71(1): 35-58.

Bergonzini JP. 2004. Changements climatiques, désertification, diversité biologique et forêts, Nogent-sur-Marne. Paris: SILVA, $98 \mathrm{p}$.

Cuni-Sanchez A, Omeny P, Pfeifer M, Olaka L, Boru Mamo M, Marchant R, et al. 2018. Climate change and pastoralists: Perceptions and adaptation in montane Kenya. Climate and Development. DOI: 10.1080/17565529.2018.1454880.

Djènontin AJ. 2010. Dynamique des stratégies et des pratiques d'utilisation des parcours naturels pour l'alimentation des 
troupeaux bovins au Nord-Est du Bénin. Thèse de doctorat, Université d'Abomey-Calavi, Abomey-Calavi, 274 p.

Dossa H, Lesse DP, Houinato M, Sinsin B, Souberou F, Yabi I. 2017. Vulnérabilité de l'élevage transhumant à la Variabilité hydroclimatique dans le Nord-Est de la République du Bénin. Revue du CAMES 8: 233-249.

Enete AA, Onyekuru AN. 2011. Challenges of agricultural adaptation to climate change: Empirical evidence from southeast Nigeria. Tropicultura 29(4): 243-249.

FAO. 2008. L'eau pour l'agriculture et l'énergie en Afrique : les défis du changement climatique ateliers régionaux. Syrte, Jamahiriya arabe libyenne, 15-17 décembre.

Feleke FB, Melaku B, Getachew G, Dana H. 2016. Determinants of adaptation choices to climate change by sheep and goat farmers in Northern Ethiopia: The case of Southern and Central Tigray Ethiopia. SpringerPlus 5: 1692. DOI: 10.1186/s40064-016-3042-3.

Gnanglè CP, Glèlè Kakaï R, Assogbadjo AE, Vodounnon S, Yabi JA, Sokpon N. 2011. Tendances climatiques passées, modélisation, perceptions et adaptations locales au Bénin. Climatologie 8: 27-40.

Graux AI. 2011. Modélisation des impacts du changement climatique sur les écosystèmes prairiaux: voies d'adaptation des systèmes fourragers. Thèse de doctorat, Université Blaise Pascal, Université d'Auvergne, France, 534 p.

Hasan MK, Kumar L. 2019. Comparison between meteorological data and farmer perceptions of climate change and vulnerability in relation to adaptation. Journal of Environmental Management 237: 54-62. DOI: 10.1016/j.jenvman.2019.02.028.

Ibrahim B, Polcher J, Karambiri H, Rockel B. 2012. Characterization of the rainy season in Burkina Faso and its representation by regional climate models. Climate Dynamics 39(6): 1287-1302. DOI: $10.1007 / \mathrm{s} 00382-011-1276-x$.

Jarawura FX. 2014. Perceptions of drought among rural farmers in the Savelugu district in the northern Savannah of Ghana. Ghana Journal of Geography 6: 102-120.

Lamb PJ. 1982. Persistence of Subsaharan drought. Nature 299: 46-47.
McDonald JH. 2009. Handbook of biological statistics, 2nd ed. Baltimore, MD, USA: Sparky House Publishing.

MEHU. 2011. Deuxième communication nationale de la République du Bénin sur les changements climatiques. Cotonou, Bénin.

Nardone A, Ronchi B, Lacetera N, Ranieri MS, Bernabucci U. 2010. Effects of climate change on animal production and sustainability of livestock systems. Livestock Science 130(1-3): 57-69. DOI: 10.1016/j.livsci.2010.02.011.

Niang I. 2009. Le changement climatique et ses impacts: les prévisions au niveau mondial. In: IEPF, eds. Adaptation au changement climatique. France: Liaison Énergie-Francophonie, pp. 13-19.

Ovuka M, Lindqvist S. 2000. Rainfall variability in Murang'a district, Kenya: Meteorological data and farmers' perception. Geografiska Annaler. Series A, Physical Geography 82(1): 107-119.

Sivakumar MVK, Maidoukia A, Stern RD. 1993. Agroclimatologie de l'Afrique de 1'Ouest: le Niger, $2^{\mathrm{e}}$ éd. Bulletin d'information $\mathrm{n}^{\mathrm{o}}$ 5. Patancheru, A.P. 502 324, Inde: Institut international de recherche sur les cultures des zones tropicales semi-arides, et Niamey-Niger: Direction de la météorologie nationale du Niger, $116 \mathrm{p}$.

Stern RD, Knock J, Grayer C, Leidi S. 2006. Instat+, Climatic guide. Univ. of Reading, Reading.

Vall E, Diallo MA. 2009. Savoirs techniques locaux et pratiques: la conduite des troupeaux aux pâturages (Ouest du Burkina Faso). Natures Sciences Sociétés 17: 122-135. DOI: 10.1051/nss/2009024.

Vall E, Lankoande YF. 2009. Perception du changement climatique des producteurs des zones de savanes agropastorales de l'Ouest du Burkina Faso et leurs pratiques d'adaptation. In : Communication présentée à la JOD (DCE Ouagadougou), le 23 octobre 2009.

Zampaligré N, Dossa LH, Schlecht E. 2013. Climate change and variability: Perception and adaptation strategies of pastoralists and agro-pastoralists across different zones of Burkina Faso. Regional Environmental Change 14: 769-783. DOI: 10.1007/s10113-013-0532-5.

Citation de l'article : Idrissou Y, Assani Seidou A, Tossou FM, Sanni Worogo HS, Baco MN, Adjassin JS, Assogba BGC, Alkoiret Traore I. 2020. Perception du changement climatique par les éleveurs de bovins des zones tropicales sèche et subhumide du Bénin : comparaison avec les données météorologiques. Cah. Agric. 29: 1. 